\title{
EDITORIAL
}

\section{Fighting Against Cancer by Integrative Medicine}

\author{
TANG Zhao-you (汤㻇猷)
}

Cancer, one of the leading causes of human death, has attracted more attention due to the complexity of etiology, diagnosis, treatment and prognosis. In the past two centuries, once the pathologist said "it is cancer", then every effort will be followed to eradicate cancer. Indeed, surgery, radiotherapy, chemotherapy and regional cancer therapies have resulted in marked improvement of cancer prognosis. With the rapid progress of molecular biology, novel treatment strategies such as molecular targeted therapy emerged, which are mainly aimed to eradicate cancer. Unfortunately, up to date, only very few cancer types had their 5-year overall survival rates exceed $50 \% .{ }^{(1)}$ It is mainly due to the inadequacy of eradication strategy, which failed to eradicate hundred percent of residual cancer, including circulating tumor cells. Therefore, debates on strategy in cancer war have recently appeared. "Trying to control the disease may prove a better plan than striving to cure it" says Gatenby RA. ${ }^{(2)}$ In the 20th century, based on the pathological background, "eradication of cancer" remained the major strategy on cancer war, substantial progress has been made, particularly for subclinical small cancers, however, metastasis and recurrence remains the obstacle for the final conquering of cancer. Therefore, besides eradication, novel strategy should be considered.

Modern Western medicine (WM) has developed rapidly since the use of microscope, and the visual field has deepened from organ, cell to molecule. Clinical oncology was developed mainly on the background of pathology. On the other hand, Chinese medicine (CM) developed through thousands years' clinical practice, and summarized into a unique theory. Based on the fact that WM and CM are just like two sides of a coin, and complementary to each other, therefore, fighting against cancer by integrative medicine might be a reasonable approach. For example, WM thinks highly of microcosmic (such as molecule) and local, whereas CM thinks highly of macroscopic and entirety; WM focuses on cancer, CM focuses on patient (with cancer); for therapeutic target,
WM emphasizes elimination of causation factor, CM emphasizes recovery from imbalance; for approach of therapy, WM stresses "to block and kill", CM stresses "to dredge"; for cancer treatment, WM aimes to eradicate cancer, whereas $\mathrm{CM}$ might be benefited from modulation of cancer as well as strengthen the host; WM usually treats one disease with one drug, and $\mathrm{CM}$ treats with multiple herbs and modifies the formula based on symptom differentiation; for assessment of therapeutic response, WM uses complete response $(\mathrm{CR})$ and partial response $(\mathrm{PR})$ based on tumor size and duration; whereas $C M$ emphasizes symptom relief, quality of life and survival; WM usually treats cancer as acute disease, and CM treats cancer as chronic disease and stresses on long-term result; for logic of thinking, WM emphasizes from theory to practice, such as making clear the cancer related molecules and developing molecular targeted therapy, and $\mathrm{CM}$ emphasizes from practice to theory, such as verifying clinically that arsenic is effective for acute promyelocytic leukemia, and eventually making clear the mechanism by binding oncoprotein, and resulting in differentiation of leukemic cell. ${ }^{(3)}$ In short, both WM and CM have their merits and demerits. Generally, WM is more powerful on cancer eradication, and CM might be of superiority on cancer modulation, particularly for minimal residual cancer after eradication therapy, and provide a hope "to give up evil and return to good", as well as another endpoint of cancer treatment- "survive with cancer".

WM and $\mathrm{CM}$ are not completely antagonistic. For example, entirety is one of the key thinking in $\mathrm{CM}$, and the recent advances in cancer research of WM also indicate that cancer is a systemic disease. It has been noted that cancer development and progression are closely related to immunity, ${ }^{(4)}$ nervous system, ${ }^{(5)}$

CThe Chinese Journal of Integrated Traditional and Western Medicine Press and Springer-Verlag Berlin Heidelberg 2012

Liver Cancer Institute of Fudan University (Zhongshan Hospital), Shanghai (200032), China

Tel: 86-21-64037187, E-mail: zytang@fudan.ac.cn

DOI: $10.1007 / \mathrm{s} 11655-012-1103-1$ 
endocrinal system, ${ }^{(6)}$ and metabolism. ${ }^{(7,8)}$ Another example, anti-inflammation (清热解毒, antipyretic detoxicate) is one of the principal treatment for cancer; in the recent decade, inflammation has also been noted to play decisive roles on tumor development and progression..$^{(9)}$ Recently, more and more studies have demonstrated that eradication therapies enhance the metastatic potential of residual cancer, even the molecular targeted therapy. ${ }^{(10,11)}$

Integrative medicine in cancer war is a novel approach to be studied. It may spend decades or even centuries to finalize. There are several integrative modes: (1) Using $\mathrm{CM}$ as the major treatment. At the very beginning, several steps may be needed. Select effective CM treatment (it is better to select a small herbal compound) in a subtype of cancer (such as arsenic in the treatment of promyelocytic leukemia) through randomized control trials, and studies on mechanism followed if clinical effectiveness was confirmed. (2) CM treatment used as adjuvant after eradication therapies of the WM, and hopefully through symptom-relief, reversing immunosuppression, minimizing metastasis, etc., and prolonging survival. (3) Using $\mathrm{CM}$ as palliative treatment for advanced cancer, and hope to provide a better quality of life and prolong survival.

In the past half century, despite tremendous work has been done in the integrative medicine on cancer war, unfortunately, it was not very successful. Studies using WM thinking, theory, criteria and models might be reasons for the failure, for example, screening Chinese herbs using animal models and criteria that used for screening of anti-cancer agents in the WM. As had mentioned above, the anti-cancer mechanism of CM may be different from that of WM to kill the cancer cell. Fighting against cancer by integrative medicine remains a difficult goal, to this end, scientists that have intimate knowledge on both WM and CM (especially the principal theory), new criteria and platform that suitable for the studies are urgently needed.

\section{REFERENCES}

1. Parkin DM, Bray F, Ferlay J, Pisani P. Global cancer statistics, 2002. CA Cancer J Clin 2005;55:74-108.

2. Gatenby RA. A change of strategy in the war on cancer. Nature 2009;459:508-509.

3. Zhang XW, Yan XJ, Zhou ZR, Yang FF, Wu ZY, Sun $\mathrm{HB}$, et al. Arsenic trioxide controls the fate of the PMLRARalpha oncoprotein by directly binding PML. Science 2010;328:240-243.

4. Schreiber RD, Old LJ, Smyth MJ. Cancer immunoediting: integrating immunity's roles in cancer suppression and promotion. Science 2011;331:1565-1570.

5. Ondicova K, Mravec B. Role of nervous system in cancer aetiopathogenesis. Lancet Oncol 2010;11:596-601.

6. Sander LE, Trautwein C, Liedtke C. Is interleukin-6 a gender-specific risk factor for liver cancer? Hepatology 2007;46:1304-1305.

7. Israelsen WJ, Vander Heiden MG. ATP consumption promotes cancer metabolism. Cell 2010;143:669-671.

8. Yecies JL, Manning BD. Chewing the fat on tumor cell metabolism. Cell 2010;140:28-30.

9. Grivennikov SI, Greten FR, Karin M. Immunity, inflammation, and cancer. Cell 2010;140:883-899.

10. Hayden EC. Cutting off cancer's supply lines. Nature 2009;458:686-687.

11. Loges $S$, Mazzone $M$, Hohensinner $P$, Carmeliet $P$. Silencing or fueling metastasis with VEGF inhibitors: antiangiogenesis revisited. Cancer Cell 2009;15:167-170.

(Received February 27, 2012) Edited by YUAN Lin 